\title{
Basic activity of Neurons in the dark during somnolence induced by Anesthesia
}

\author{
Etindele Sosso FA ${ }^{1,2 *}$, Hito $\mathrm{MG}^{3}$ and Bern $\mathrm{SS}^{4}$ \\ ${ }^{1}$ Research Center in Neuropsychology and Cognition, Quebec, Canada \\ ${ }^{2}$ Department of Biological Sciences, University of Montreal, Quebec, Canada \\ ${ }^{3}$ Department of Psychology and Sports Science, Justus-Liebig University, Giessen, Germany \\ ${ }^{4}$ Department of Fundamental Neuroscience, University of Lausanne, Switzerland
}

*Corresponding author: Faustin Armel Etindele Sosso, PhD, Research Center in Neuropsychology and Cognition, Quebec, 90, avenue Vincent d'Indy, H2V2S9, Canada, Tel: +1 514343 6111\#3187; E-mail: faustin.armel.etindele.sosso@umontreal.ca

Received: June 15, 2017; Accepted: June 27, 2017; Published: June 30, 2017

Citation: Etindele Sosso FA, Hito MG, Bern SS (2017) Basic activity of neurons in the dark during somnolence induced by anesthesia. J Neurol Neurosci 8:3.

\section{Abstract}

Aim:The first experience modulates the network of integration and memory in the sleeping cortex. Depending on the characteristics of this initial stimulation, the brain adjusts his electrical activity to respond during wake or sleep. This adjustment is different for every sub type of neurons and are influenced by the information drove by stimuli. Pyramidal cells and inhibitory cells behaviours after a short reminder during somnolence or sleep deprivation are still unclear.

Materials and methods:Inhibitory interneurons and excitatory pyramidal cells in the primary visual cortex of anesthetized mice were stimulated in the dark after sleep deprivation of 12 hours. Analysis of electrical activity of sub-populations of neurons were performed with electrophysiological recordings.

Results: A primary stability was revealed in the firing pattern. In the global sample, the discharge of inhibitory interneurons was the same before and after visual stimulation with induced-anesthesia somnolence. Absence of orientation in the stimuli didn't affected both inhibitory and excitatory activity. The findings also suggested the interplay between primary visual cortex and the short-term plasticity enhances during sleep.

Conclusion:The conditions of the neurotransmission during wake or first stage of sleep prepares the mechanism of sleep-dependent plasticity. Stimuli properties affects the perception and memorization of new information, but the consolidation of their information is altered during sleep deprivation.

Keywords: Sleep; Somnolence; Inhibitory interneuron; Excitatory pyramidal cell; Neuronal activity; Plasticity, Learning

\section{Introduction}

After many decades of research in the vision field, it is not remained clear how shapes and objects inside visual stimuli are encoded and memorized by the brain. The recent evidence demonstrated that the visual system drives original information of an image, and the persistence of this trace on the neuronal memory is ensured by V1 [1]. The continuous effects of the stimuli are correlated with memorization, while trace remains in the cortical circuitry for few seconds [2]. The truth is, visual stimuli composed of many parameters, can affect the behaviour of cortical neurons. These parameters like spatial frequency, shape of target or temporal frequency [3-5]; induced modification of activity. The brain received continuously inputs from the time eyes are open until individuals sleep [6]. This activity is expressed with number of spike generated during a period, through all the brain or higher specialize areas. The firing rate becomes an important measure of changes in the dynamic of central nervous system, especially for the electrical activity in the primary visual cortex. It was already reported which kinds of effects a stimulus with an orientation has on primary visual cortex, as well as spatial frequency and size of dots and gratings [4,7]. But effect of visual stimulation without orientation on this structure is laking accuracy. Another question is how integration is made, in the different types of sub neurons. The present investigation focus on fast spike interneurons behaviour, when they are stimulated with some stimuli without an orientation to drive encoding. It is not reported to our knowledge, if a stimulus without orientation changes the neuronal activity of primary visual neurons, especially if combining with another stimulus. In the following paper, effects of a short stimulus on the visual neurons behaviour to succeeding stimuli are tested. The objective is to verify if the neurons modified their electrical activity while keeping a trace of the other grating's parameters, and if this synaptic rewiring is sleep or dark dependent. 


\section{Methods}

\section{Ethics statement}

All surgical and experimental procedures were approved by Justus-Liebig University Institutional Animal Care and Use Committee and were in accordance with National Institutes of Health guidelines. Male C57BL/6 mice were housed in a room with a 12:12-h light-dark cycle for one week before the first surgery and remained on this cycle throughout. All training and experiments were performed during the light period at approximately the same time of day (2-h range) for each mouse. Mice were allowed food and water ad libitum while in their home cages.

\section{Animal preparation}

30 white C57BL/ 6 mice (young adult's male) aged from 8 to 11 weeks were employed in this investigation. Rimadyl $(1 \mathrm{mg} / \mathrm{ml})$ was injected to induce anesthesia. The effects were extended with $3 \%$ isoflurane mixed with medical-grade oxygen and then maintained at $1-2 \%$ isoflurane throughout procedures. Each mouse was injected with $0.15 \mathrm{ml}$ of Rimadyl (1 $\mathrm{mg} / \mathrm{ml}$ subcutaneously) and installed in a stereotaxic equipment. The skull's surgery was performed by scalping the crown and removing the fascia and scored with the tip of a scalpel blade. After the skull and the bone had been cleaned, dried, and leveled; the recording chamber was installed so that its center was at a stereotaxic position corresponding to V1 and glued in place. Many layers of low-viscosity cyanoacrylate were applied over the surface of the skull after the surgery. An initial layer of Metabond (Parkell) was applied over the cyanoacrylate, before fixed the recording chamber.

\section{Experimental setting}

In vivo electrical activity of the brain was recorded with two microelectrodes (small electrodes in glass, filled with $0.9 \%$ $\mathrm{NaCl}$ ) directly in $\mathrm{V} 1$, not deeper than $1 \mathrm{~mm}$. The signal recorded by each electrode was converted and encoded after filtering (with the software Spike 2, Cambridge Electronic Design, CED Limited, Cambridge, England), and exhibited on the computer. Then, neurons inside the multi-unit signal were dissociated off-line with the spike sorting technique. Firing rate of each neuron was correlates with its waveform, and display in a cluster. The firing rate regularity of all these neurons was confirmed qualitatively by visual control. For each isolated cell, cluster classification was carried out until a complete separation appeared between each cluster. The segregation was characterized by a chosen $\mathrm{K}$ mean value of 2.5 (size of each cluster), ensuring an absence of contamination between neighbouring cells or noise in the system. To be considered, cluster should not have any overlap with another, to be considered as authentic. For the whole project, visual stimuli were used. They were generated with Matlab (g.STIMunit realtime visual stimulation unit) Visual stimuli were shown on a 16.5-inch Monitor, set at a distance ranging from 26 to $30 \mathrm{~cm}$ from the mice's eye. Temporal frequency was equal to 0.5 Hertz, spatial frequency was 0.3 cycles/0, contrast was $100 \%$ and phase was 0.5 . These characteristics were identical during all the experiments.

Five orientations $(0,45,90,135$ and 180 degrees) spaced with 45 degrees, were selected and used for the study. Control orientations were displayed in a random sequence. Each oriented stimulus was presented in blocks of 25 trials lasting $4.1 \mathrm{~s}$ each with a random inter-trial interval $(250 \mathrm{~ms}-2 \mathrm{sec})$ during which, no stimulus was presented. Once these control orientations tuning curves were characterized, the following protocol was tested: a briefly applied grating (BAG, a short stimulus with a $250 \mathrm{~ms}$ duration), followed without delay, by a control grating (CG, duration: $4 \mathrm{~s}$ ) where neuronal responses were measured. Designed as the control grating, the briefly applied grating was a dot stimulus [8], composed of four thousand white dots with no orientation, appearing on a black background during $250 \mathrm{~ms}$. Each block of BAG + CG has the same random interval ( $250 \mathrm{~ms}-2 \mathrm{sec}$ ) between BAG and CG, during all the protocol. The stimulus properties were same in either case. The orientation tuning curves were investigated prior to-and following BAG. In this investigation, white dots were displayed on a black background during $250 \mathrm{~ms}$. The length was chosen because it is close to human saccades.

\section{Statistical analysis}

Using Matlab tools box (Data Analysis and Statistics unit) the evoked responses were extracted from recorded signals and transformed into numerical values, expressing firing rates of neurons. Since orientation tuning is best described with Gaussian-like functions in neuroscience [9], raw data was fitted with the Von Mises function. This procedure was used to determine the preferred orientation of each cell, and calculated shifts in orientation preference. Like Swindale et al. the Von Mises function was used and defined as: $y=b+$ $r m x^{*} \exp \left(-\left((x-x 0)^{\wedge} 2\right) / 2 s^{\wedge} 2\right)$ with: $b=$ base, $r m x=m a x$ firing rate, $\mathrm{x}=$ orientation test, $\mathrm{x}_{0}=$ optimal orientation, $\mathrm{s}=$ sigma [9]. To compute the firing rate, it is a mean of cells discharge after each presentation of the stimuli BAG+OTG. So, for each orientation of the stimuli, it was obtained a mean of 25 firing rate. Normality of the distribution was tested with shapiro-wilk test.

\section{Results}

The visual stimulation protocol was applied: a briefly applied oriented grating which had no angle of orientation, because composed of four thousand white dots; display on a black background during $250 \mathrm{~ms}$. This BAG was automatically followed by a control grating (CG, duration: 4 seconds). The total sample size was 200 cells. 50 cells $(25 \%)$ with an Orientation Selectivity Index [10] $<0.3$ (value of the exclusion threshold) were excluded from the analysis of the current study. The results were obtained from a total population of 150 cells. Figure 1 shows the distribution of excitatory cells and Inhibitory interneurons in the population. Figure $\mathbf{2}$ exhibits examples of two neurons with their firing rates before (control) and after the visual stimulation. Figure 3 exhibits the distribution of global firing for the entire sample, before and 
after BAG. An orientation selectivity index of 0.3 was calculated [10] by dividing the firing rate at orthogonal orientations (baseline of the tuning curves) by the firing rate for the preferred orientation, and subtracting the result of 1 . The closer the OSI is to 1 , the stronger is the orientation selectivity. So, all cells with an OSI under 0.3 were excluded from our analysis, as we choose this 0.3 as threshold to exclude broadly tuned cells. Descriptive statistics were applied to the interneuron's population to investigate changes in the electrical activity after BAG. For control and post stimulation, we compared intensity of response (for their preferred orientation and orthogonal orientation), before and after BAG. Findings revealed the same behaviour for control and post stimulation. The firing rate pattern was similar.

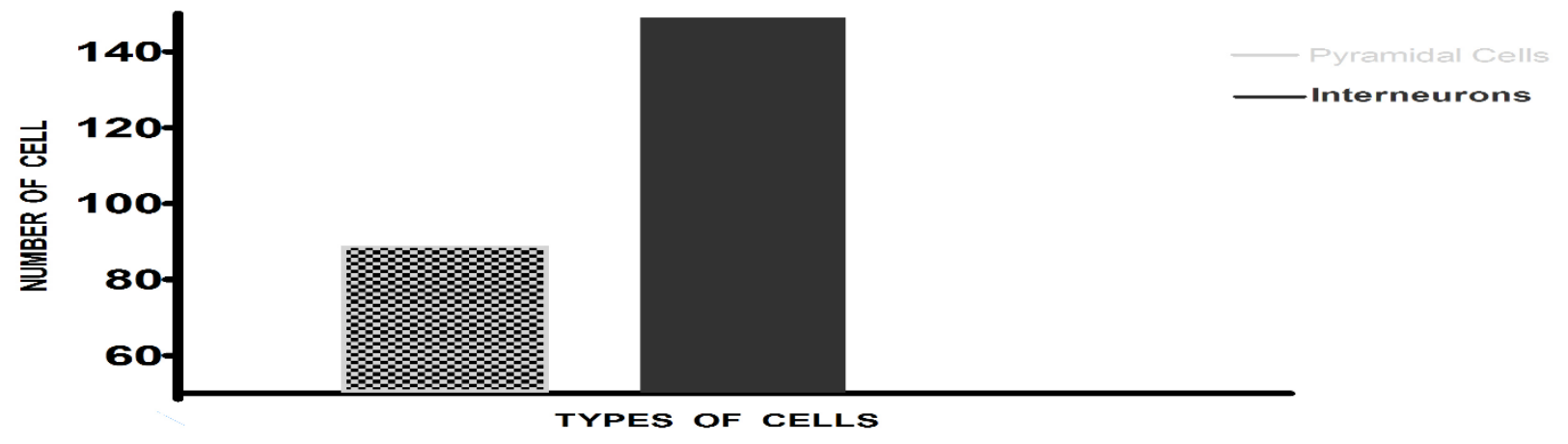

Figure 1 Distribution of Pyramidal cells and inhibitory interneurons.

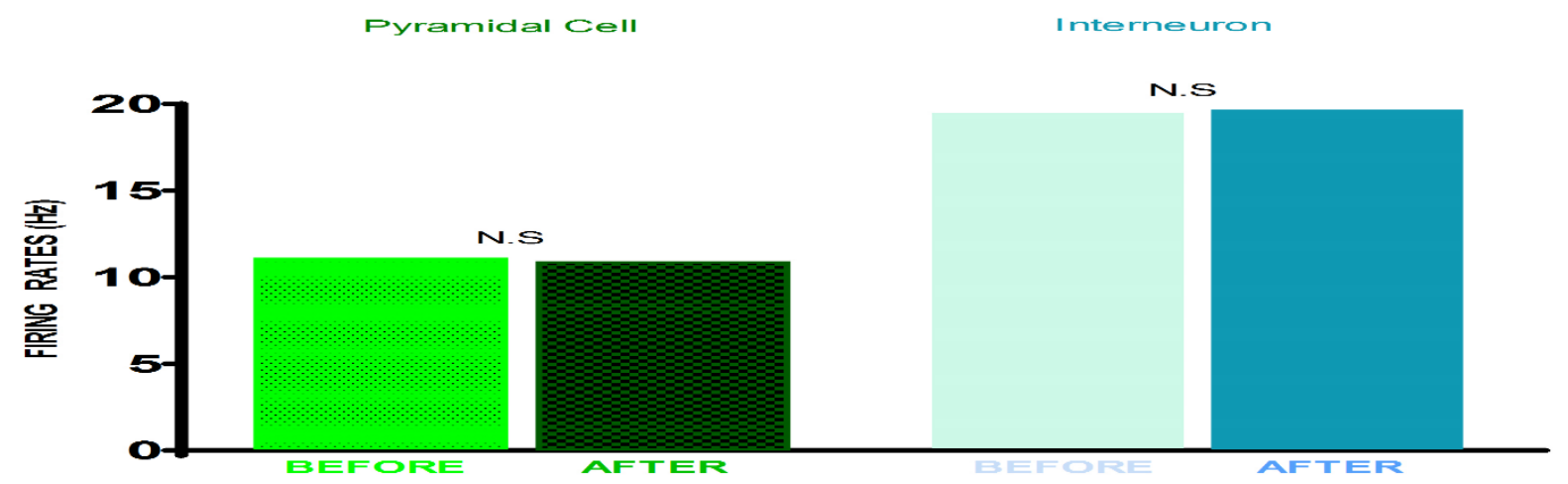

Figure 2 Examples of pyramidal cell and interneuron with their firing rate before and after induced somnolence.
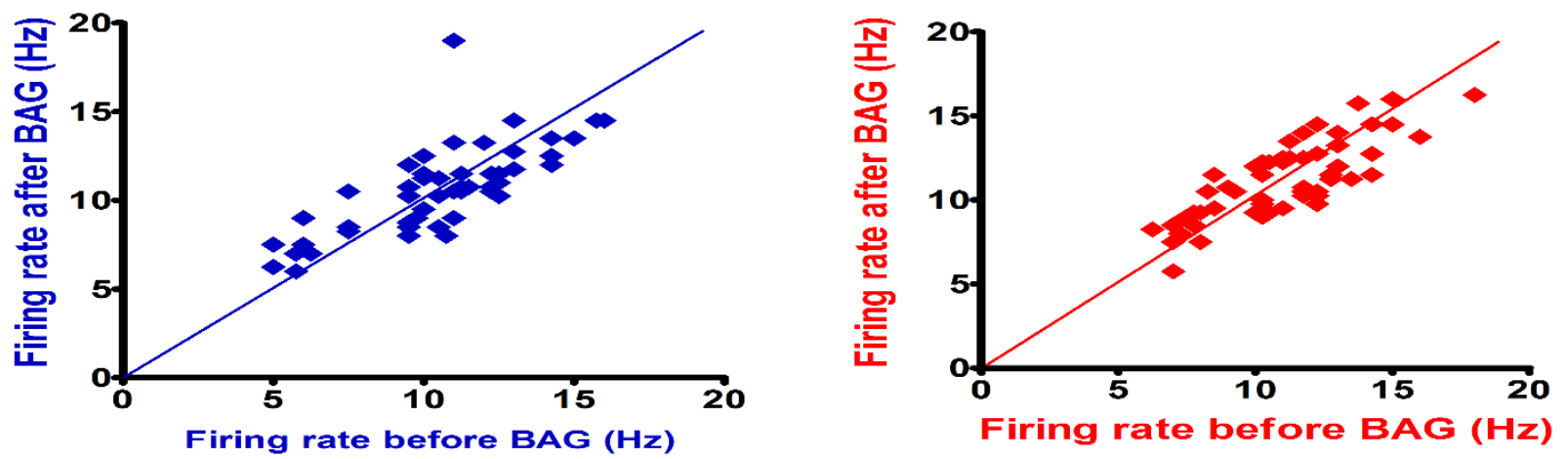

Figure 3 General pattern of discharge before and after recordings in the dark. blue graph (left) represents interneurons and red graph (right) represents pyramidal cells.

\section{Discussion}

In the present research, many measures have been employed to ensure the validity of findings and an accurate control of potentials bias. First, the visual stimulation was made with a homemade Matlab script. The program was run with Matlab Software. This software synchronized stimuli and recordings, and avoid human interventions during the 
experiment because all the procedure was automated. Matlab also has benefits compared to others programming languages, to be easily editable off-line or online; and to have significant less bug or compatibility issues than most tools on the market. There is no influence of anesthesia on the neuronal activity, because the chosen mixture of Rimadyl and isoflurane have to our knowledge no interaction with the memory process. This condition ensures there is no interaction with attention and, the output values are not corrupted. It has a long delay before action compared with Ketamin or xylaxine, but his action in longer without any effects on heart rhythm or brain consummation of oxygen. Xylocaine was only injecting before surgery to avoid a silent cortex due to micro infiltration. LFP were filtered before the recordings. All the experiments were made in the dark, to reduce noise and extra perception of light or white screen. Even if the visual stimuli were displayed contralateral, there is no deprivation of the second eye. This additional control was used in previous studies, to maintain the natural visual acuity and not to alter single-unit activity. Our results revealed a perception mechanism of interneurons, which is correlated with absence of orientation. Our findings showed that, the visual induced-memory is dependent of interneuron's activity. if the stimuli include no attribute, integration is altered because the pathway of plasticity is disturbed. A target display to eyes, leaves a trace in the neuronal structures involved in memory [1,2]. In line with previous research, our findings confirm implication of orientation in the discharge of cells. [11,12], and showed that stimuli missing orientation; does not change significantly the responses of interneurons after the second stimulus. Discharge depends strongly of the influence of the second grating with an orientation. Even if a stimulus created a change in spiking, an orientation is essential to induce neuronal plasticity [13] The present research confirmed the hypothesis stating that, the predictiveness of a stimulus affects the amplitude of neuronal responses [14]. Prediction is like learning, and neurons learned faster with repetitive stimulus display with the same orientation remaining unchanged, associate to a combination of parameters included inside daily inputs drives by retina and visual areas. It is also coherent with studies stating the complex combination of different kinds of stimuli (environmental, physical, drugs, family medical history, etc.), can strongly affect the brain activity [6,15-17]. The findings of this paper question the true role of stimuli's properties, in the responses of neurons. The short-term plasticity and the temporary neural suppression (necessary for maturation of the brain, are directly concerns by these unrevealed mechanisms [18].

\section{Conclusion}

Initial perception prepared the cortex to integrate the following information. Without sensitive properties of environmental stimuli enhancing encoding processing, the stimuli's capacity to modify neuronal activity is reduced, as well as the recognition and consolidation in the neuronal circuitry of new information. The short-term plasticity is correlated with amount of additional information received, as well as the intensity of firing which is an expression of the balance excitation-inhibition. Interneurons and Pyramidal neuron's activities are maintained stable, suggesting their activity is associated with a specific pattern of discharge during somnolence or beginning of sleep.

\section{Author contributions}

ESFA, HMG and BSS designed the study. HMG and BSS performed experiments and analysis. ESFA, HMG and BSS interpreted results of experiments. ESFA drafted manuscript and figures. ESFA, HMG and BSS edited and revised the manuscript. ESFA, HMG and BSS approved the final version of the manuscript.

\section{References}

1. Greene E (2016) Information persistence evaluated with lowdensity dot patterns. Acta psychologica 170: 215-225.

2. Bruchmann M, Thaler K, Vorberg D (2015) Visible persistence of single-transient random dot patterns: Spatial parameters affect the duration of fading percepts. PloS one 10: e0137091.

3. Ohnishi Y, Kawano K, Miura K (2016) Temporal impulse response function of the visual system estimated from ocular following responses in humans. Neu res.

4. Ringach DL, Mineault PJ, Tring E, Olivas ND, Trachtenberg JT, et al. (2016) Spatial clustering of tuning in mouse primary visual cortex. Nat Commun 7: 12270.

5. An X, Gong H, Mcloughlin N, Yang Y, Wang W (2014) The mechanism for processing random-dot motion at various speeds in early visual cortices. PloS one 9: e93115.

6. Etindele SFA, Raouafi S (2016) Brain disorders: Correlation between cognitive impairment and complex combination. Ment Health Fam Med 12 :215-222.

7. Tory M, Swindells C, Dreezer R (2009) Comparing dot and landscape spatializations for visual memory differences. IEEE Trans Vis Comput Graph 15: 1033-1039.

8. Bagcchi S (2017) Looking back at yellow fever in Angola. Lancet Infect Dis 17: 269-270.

9. Reimann MW, King JG, Muller EB, Ramaswamy S, Markram H (2015) An algorithm to predict the connectome of neural microcircuits. Front Comput Neurosci 9: 120.

10. Crane RJ, Berkley JA (2017) Progress on growth faltering. Lancet Glob Health 5: e125-e126.

11. Hutchinson CV, Ledgeway T, Allen HA (2014) The ups and downs of global motion perception: a paradoxical advantage for smaller stimuli in the aging visual system. Front Comput Neurosci 6: 199.

12. Pageau M, De Guise D, Saint-Amour D (2015) Random-dot stereopsis in microstrabismic children: stimulus size matters. Optom Vis Sci 92: 208-216.

13. Kumano H, Uka T (2013) Responses to random dot motion reveal prevalence of pattern-motion selectivity in area MT. J Neurosci 33: 15161-15170.

14. Le Pelley ME, Vadillo M, Luque D (2016) Learned predictiveness influences rapid attentional capture: Evidence from the dot probe task. J Exp Psychol Learn Mem Cogn 39: 1888-1900.

15. Etindele Sosso FA (2017) Sleep disorders and insomnia: effects on a young population. Psychology and Psychiatry 2: 26-32. 
16. Etindele-Sosso FA (2017) Neurocognitive game between risk factors, sleep and suicidal behaviour. Sleep Science 10: 41-46.

17. Etindele Sosso F, Raouafi S (2016) Appropriate sleep uration and physical activity modulate cognitive improvement. J Sleep Disor: Treat Care 5: 4.
18. Etindele Sosso FA (2017) Visual dot interaction with short-term memory. Neurodegener Dis Manag. 